\title{
Heuristic Solutions Related to Challenges of Optical Burst Switching Paradigm
}

\author{
Dr. Amit Kumar Garg \\ Professor \& Head \\ Department of Electronics and Communication Engineering, MMEC \\ M M University Mullana, Ambala (India) \\ garg_amit03@yahoo.co.in
}

\begin{abstract}
Several optical network switching paradigms have been under intensive research to support rapidly increasing bandwidth requirements. Of all these paradigms, optical burst switching (OBS) is a promising switching paradigm for the next-generation Internet infrastructure. Despite the significant contributions of OBS to high speed networks such as dynamic connection setup and strong separation between data and control plane, there are several issues that are to be resolved because the present OBS research studies have many differences and thus need further study. In this paper, some of author's own past researches have been summarized to overcome few issues of OBS; related to network architectures, contention, burst assembly, congestion, complex scheduling schemes, quality of service (QoS), burst dropping, routing etc.
\end{abstract}

Index Terms — optical burst switching, burst assembly, wavelength reservation, burst dropping, routing.

\section{INTRODUCTION}

Presently, Optical Burst Switching (OBS) technology [1-2] is under study as a promising solution for optical Internet backbone in the near future, since OBS eliminate the electronic bottleneck at switching node with the help of no O/E/O conversion and guarantee the class of service without any buffering. The OBS network can be envisioned as two coupled overlay networks: a pure optical network transferring data bursts and a hybrid control network transferring control header packets. The control network is just a packet switched network, which controls the routing and scheduling of data bursts in the all optical network based on the information carried in their control header packets. This coupled overlay networks take advantage of both mature electronic control technologies and promising optical transport technologies. OBS aims to provide higher utilization and flexibility than the current optical paradigms of multiple optical-electronic- optical conversions.

\section{OPTICAL BURST SWITCHING AND RELATED ISSUES}

OBS has been considered as a middle term solution towards all optical packet switching whose goal is to improve wavelength utilization and sharing by introducing a dynamic wavelength management. In OBS networks, data never leave the optical domain: for each data burst assembled at the network edge a reservation request is sent in advance as a separate control packet. There are two kinds of nodes: edge and core routers. The main function of edge nodes is the burst assembly: these nodes are the gateways between traditional "electrical" 
networks and high speed optical networks; they must collect IP datagram and assemble them into burst according to proper assembly algorithms. Core nodes, on the other hand, deal with optical data bursts and the related control packets. They have to set up on the fly internal optical paths for switching bursts and take them hop-by-hop closer to their final destination. In addition, the offset time allows the core router to be buffer-less, avoiding then the employment of optical memories, e.g. fiber delay lines, required on the contrary by optical packet switching. The control packet carries relevant forwarding information, as the next hop, the burst length and the offset time. It precedes the data burst by a basic offset time that is set to accommodate the non-zero electronic processing time inside the network and dynamically set up a wavelength path whenever large data flows are identified and need to traverse the network. Only the control packet is converted between optical and electronic domains, therefore is the only information delayed because of the conversion. Optical Burst switching schemes differ based on how and when the network resources like bandwidth, are reserved and released. In case of an immediate reservation scheme such as Just in time (JIT), an output wavelength is reserved for a data burst immediately after the arrival of the corresponding control burst; if a wavelength cannot be reserved at that time, then the setup message is rejected and the corresponding burst is dropped. In a delayed reservation scheme such as Just enough time (JET), the control burst (CB) and the data burst (DB) are separated in time by an offset value in order to accommodate the processing of the $\mathrm{CB}$. An output wavelength is reserved for a burst just before the arrival of the first bit of the burst. If, upon arrival of the setup message, it is determined that no wavelength can be reserved at the appropriate time, then the setup message is rejected and the corresponding burst is dropped. In JET, the size of the burst is decided before the CB is transmitted by the source. The offset between $\mathrm{CB}$ and DB is also calculated based on the hop count between the source and destination. At each node, if bandwidth is available, the CB reserves wavelength for the upcoming burst for a fixed duration of time. The reservation is made from the time when the first bit of DB reaches the node till the last bit of DB is transmitted to the output port. This eliminates the wavelength idle time which is the main difference between JET and JIT. Since the wavelength is reserved for a fixed duration, there is no need for explicit signal for releasing the reserved wavelength along the path. Since there is no wastage of bandwidth in this scheme, the network utilization for this scheme is higher than with the other schemes. Also, JIT is simpler to implement as compared to JET. Thus, the_most used reservation protocol in OBS network is Just-Enough-Time (JET) [1]. JET is a delayed reservation protocol which allows reserving a wavelength channel just for the burst duration, starting at the predicted burst arrival time. If the reservation is successful, the control packet is forwarded to the next node; otherwise the correspondent burst is blocked. The use of this reservation protocol doesn't allow to fully utilizing the bandwidth: in every channel there is portion of unused bandwidth between bursts that have made a reservation, called void. In order to get a good utilization of the available resources, an efficient reservation process is required. To this end, effective scheduling algorithms have to be developed [2]. An optical burst consists of assembled IP packets directed to the same destination address. Burst assembly process starts at the initial packet arrival and continues for the period of burst assembly time at an edge node in OBS networks. The length of burst assembly time is allowed to be fixed or variable according to the design of OBS nodes. "Burst buffering delay" occurs through the period between the arrival of initial IP packet and the transmission of a burst. The optical burst is buffered during the burst assembly process and it further waits for offset time after the burst is completed. Burst buffering delay is large because of offset time and burst assembly time. Therefore efficient burst assembly mechanism with delay reduction for the burst buffering delay is desired.

A major concern in OBS networks is contention and burst loss. Typically, there are two main sources of burst loss: contention on the outgoing data channels and contention on the outgoing control channel. Contention is aggravated when the traffic becomes bursty and when the data-burst duration varies and becomes longer. Contention and loss may be reduced by implementing contention resolution policies. There are different types of contention resolution techniques, such as time deflection (using buffering), space deflection (using deflection routing) [3-4], wavelength conversion (using wavelength converters) and soft contention resolution (using different contention resolution algorithms). Researches have proposed some more dropping policies, such as tail 
dropping and head dropping. The limitation of such policies is that they cannot utilize bandwidth efficiently. Thus, critical design issue in OBS is finding ways to minimize burst dropping resulting from resource contention.

Efficient admission control and resource provisioning mechanisms are needed to support QoS. Several schemes have been proposed to support the relative service differentiation in OBS. Though it has been well accepted that absolute QoS support is important, there is no scheme in the literature to provide absolute QoS in OBS [5-6].

OBS has received considerable attention in past few years and various solutions have been proposed and analyzed in an attempt to improve its performance. The following sections describe the various OBS performance oriented optimal solutions for resolving its issues related to OBS architecture, channel utilization, burst assembly, reservation scheme, burst contention, bandwidth utilization etc.It is seen that the published results are better in terms of lower blocking probability, better utilization of bandwidth and resources, improved throughput and better traffic control . Finally, concluding remarks have been presented.

\section{NOVEL OBS ARCHITECTURE}

In [7], a novel optical burst switching (OBS) high speed network architecture has been proposed. To verify its feasibility and evaluate its performance, just-enough-time (JET) signaling has been considered as a high performance protocol. In the proposed architecture, to avoid burst losses, firstly, a short-prior confirmationpacket (SPCP) is sent over the control channel that simulates the events that the actual packet will experience. Once SPCP detects a drop at any of the intermediate nodes, the actual packet is not sent but the process repeats. In order to increase network utilization, cost effectiveness and to overcome some limitations of conventional OBS, optical label processing utilizing inherent codes (e.g., orthogonal optical codes (OOC)), which are codified only in intensity, has been used. Optical label processing consists of optical correlation of the OOC labels that arrive in the OBS node. Through simulation results obtained, it is shown that a decrease in burst loss probability, cost effectiveness and a gain in processing time are obtained when optical label processing is used as compared with electronic processing.

Considering that up to $50 \%$ of IP traffic consists of packets smaller than 520 bytes, then for a system of 10Gbps bit rate, packets with duration of $0.417 \mu$ s are considered, $50 \%$ of these packets have the mean length of 33 ns. Then by using optical label processing, OBS network approaches conventional packet switching granularity and thus network utilization can be increased especially for small bursts composed by IP traffic. The effects of bit rate and minimum burst length of different number of wavelengths for transport by OBS network are shown in Fig. 1 (a), for both optical and electronic label processing. It has been observed that when optical label processing is utilized, in comparison with electronic processing, the minimum burst length decreases for all number of wavelengths considered. In Fig. 1(b), it is seen that the utilization efficiency for optical label processing is higher than that for electronic processing. However, when the processing time is higher than the burst length, the utilization efficiency of electronic label processing decreases whereas the utilization efficiency of optical processing is practically the same for different bit rates. These results are obtained by considering the burst loss probability equal to zero. This is an effective solution for adjusting burst length. 


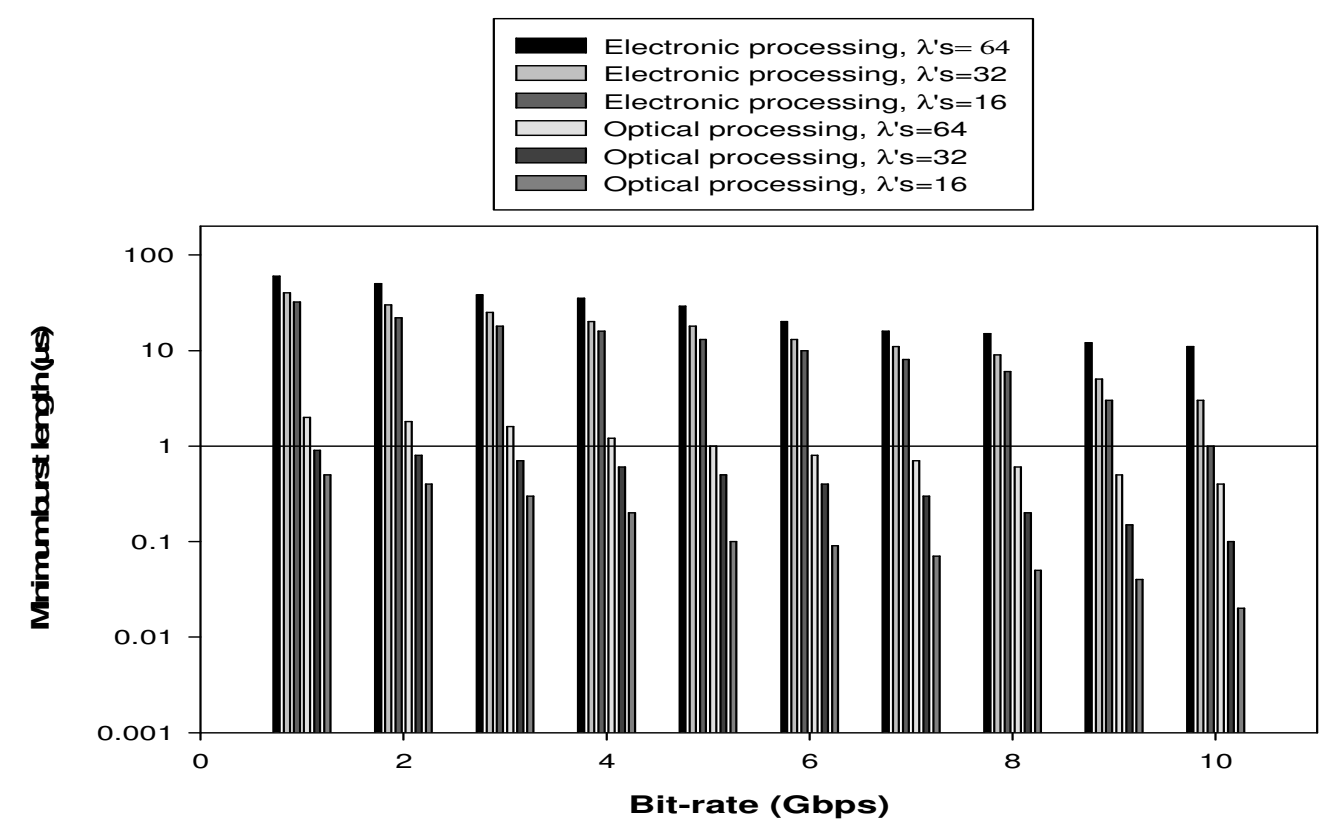

Fig. 1: (a) Minimum burst length Vs bit rate as a function of number of wavelengths

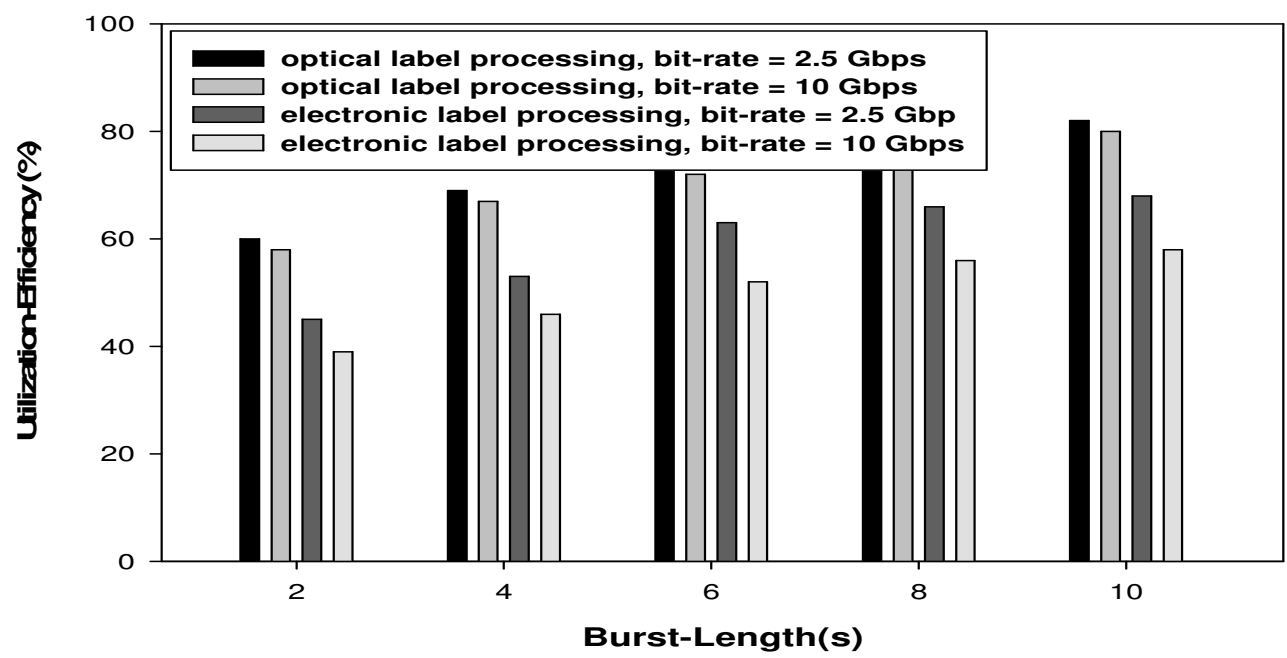

Fig. 1: (b) Utilization efficiency Vs the burst length as a function of bit rate.

Figure 2 shows the result of throughput of the network versus load. It has been observed that the throughput value of proposed OBS architecture is higher than conventional OBS (JIT-based) architecture. This is attributed as throughput gain, which provides an improvement in wavelength usage of OBS network. And the improvement in return increases the network utilization gain. Thus the proposed OBS architecture can use bandwidth more efficiently. 


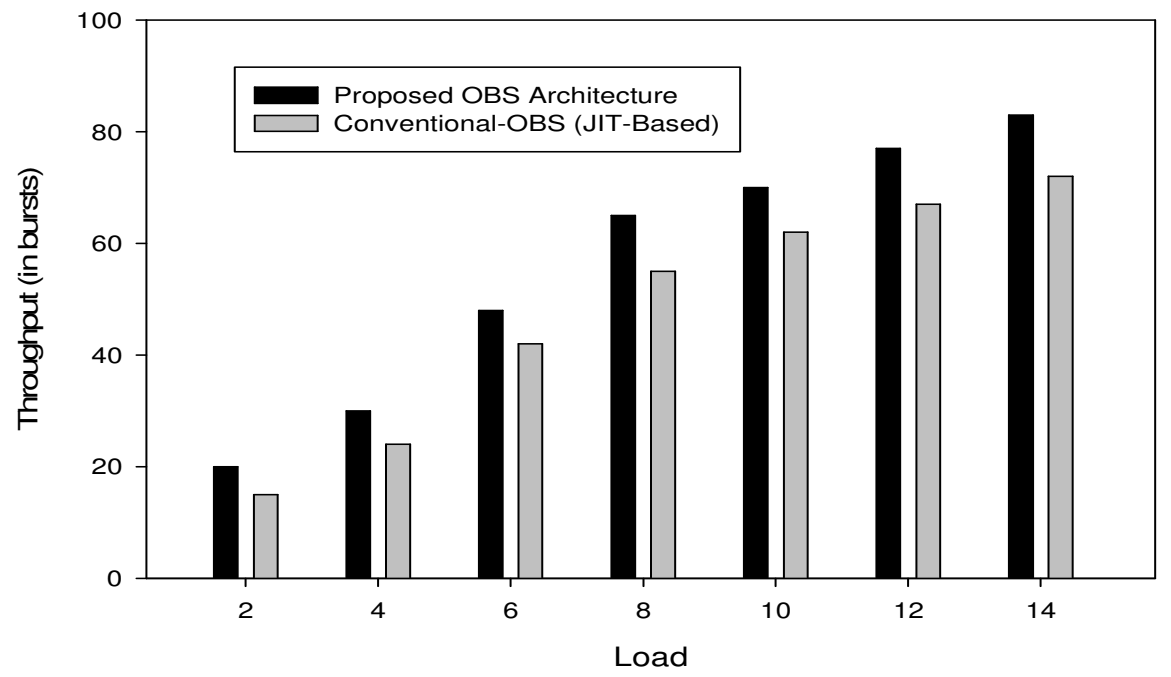

Fig.2: Throughput Vs load

Considering that the average burst length is strongly related to the performance of the OBS control channel, optical code labels and optical processing have been applied to make the JET signaling at the OBS control channel. From Table 1, it has been observed that for a 10-Gbps bit rate the minimum burst length accepted to be transported is $15 \mathrm{~ns}$ and $4 \mu$ s for optical and electronic label processing, respectively.

Table 1: Minimum burst length required for electronic and optical label

\begin{tabular}{|c|cc|}
\hline Processing-Time & \multicolumn{2}{|c|}{ Minimum Burst-Length } \\
\hline & \multicolumn{2}{|c|}{ (Bit-rates) } \\
& $2.5 \mathrm{Gbps}$ & $10 \mathrm{Gbps}$ \\
\hline Optical (ns) & 60 & 15 \\
\hline Electronic( $\boldsymbol{\mu s})$ & 13 & 4 \\
\hline
\end{tabular}

Further, OBS network performance has been performed as a function of time processing and burst length, the performance metrics such as network utilization efficiency $\left(\eta_{N U E}\right)$ and economy of wavelength (EW) are given by:

$$
\eta_{N U E}(\%)=\frac{T_{\text {Burst }}}{T_{\text {Burst }}+T_{P}} \times 100 \text { and } E W(\%)=\frac{W_{\text {Electronis }}-W_{\text {Optical }}}{W_{\text {Electronic }}} \times 100
$$

( $T_{\text {Burst }}$ : burst-length; optical label processing time $\left(T_{p}\right) ; W_{\text {Electronic }}$ and $W_{\text {Optical }}$ : numbers of wavelengths utilized for electronic and optical label processing, respectively).

There are 54 wavelengths at 10 Gbps for data and one control wavelength at 2.5 Gbps in every link of the network. Figure 3 shows that the burst loss probability for optical label processing is lower than electronic 
processing at same traffic-load (0.6 Erlang).Using electronic label processing, a burst-loss probability of $1 \times 10^{-}$ ${ }^{3}$ (approx.) is obtained for 33 and 60 wavelengths (with BAR = 3200 and 6400 Bps) whereas 30 and 53 wavelengths are required for optical label processing. This shows an improvement in wavelengths usage of OBS network, which in turn increases the network utilization gain (efficiency). For the burst loss probability of $1 \times 10^{-}$ ${ }^{3}$ (approx.), there is an economy of wavelength (EW) equal to 9 and $12 \%$ at BAR of 3200 and 6400 Bps, respectively.

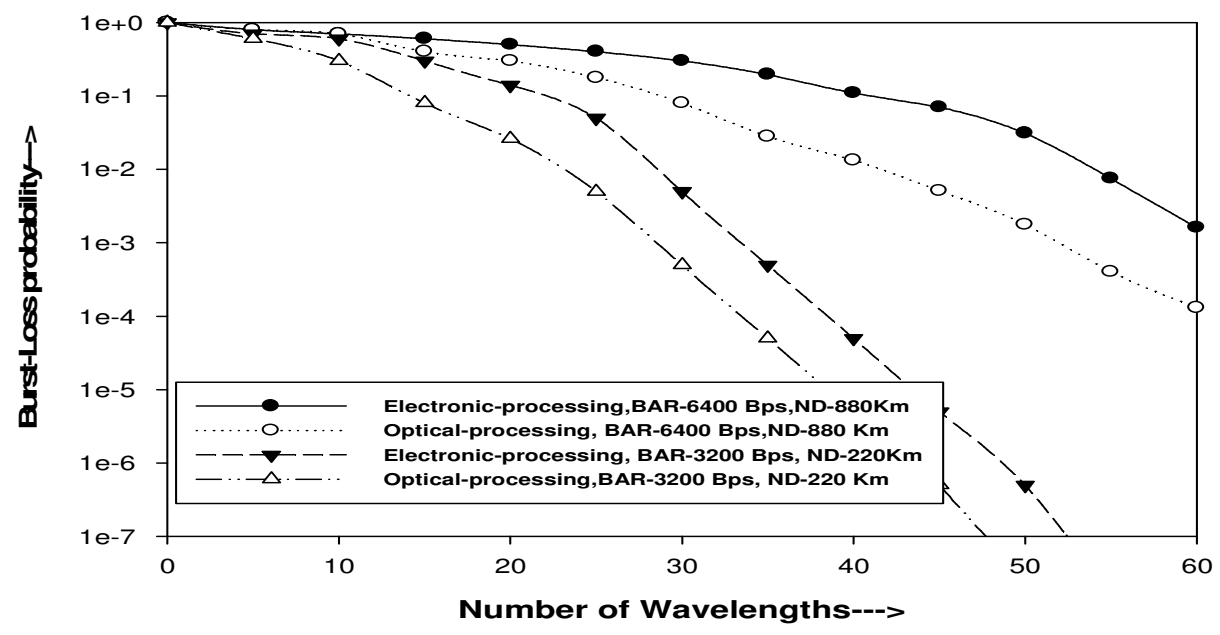

Fig.3: Burst-loss probability Vs number of wavelengths

\section{OPTIMIZING CHANNEL UTILIZATION}

In order to make OBS a viable solution, the wavelength scheduling algorithms need to be able to utilize the available wavelengths efficiently, while being able to operate fast enough to keep up with the burst incoming rate. Unfortunately, horizon scheduling cannot utilize the voids created by previously scheduled bursts, resulting in low bandwidth utilization. To date, minimum starting void (Min-SV) is the fastest scheduling algorithm that can schedule wavelengths efficiently. However, its complexity is $\mathrm{O}(\log \mathrm{m})$ where ' $\mathrm{m}$ ' is the number of voids per channel and it requires $10 \log (\mathrm{m})$ memory accesses to schedule a single burst. This means that it can take upto several microseconds for each burst request, which is still too slow to make it a practical solution for OBS deployment. In [8], an efficient scheme has been proposed for optimizing channel utilization in OBS networks. In the proposed approach, a burst is represented by an interval of time. The process of scheduling a number of bursts, thus, turns to be a process of fitting a set of the corresponding time intervals on a channel time line that represents a channel-time resource. By doing so, the scheduling process can be formulated as a combinatorial optimization problem. Then, graph theory is applied to schedule as many non-overlapping intervals as possible onto the channel time line. The underlying concept of the proposed scheduling scheme is that of by delaying the scheduling of a burst, a much better decision can be made about a number of bursts all-together. Fiber Delay Lines (FDLs) can provide a limited time delay, the amount of delay is determined by the length of the FDL. In the OBS systems that do not have FDLs, data has to be discarded if it cannot be forwarded to the desired output at the time of arrival. This scheme is shown, through simulations, to improve performance in terms of burst loss probability, channel utilization, fairness-control and data throughput over existing schemes. Thus the proposed scheme is well suited for high performance networks in terms of reliability. 
Figure 4 compares the burst-loss probability of the proposed scheduling with that of the JIT-scheduling. Both probability distributions of the source "on" and "off" states are exponentially distributed. The results show that the proposed scheduling outperforms the JIT scheduling over the entire range of the mean offered load. The gain increases slightly with load. Better improvement in burst loss probability has been achieved, particularly in the full load to overload region.

It is observed that burst loss probability is high in JIT scheme as compared to Heuristic scheme because JIT is immediate reservation where as Heuristic scheme is delayed reservation. It has been observed that in optical label processing alongwith delayed reservation as compared with electronic processing utilizing immediate reservation, the minimum burst length decreases for all number of wavelengths (e.g. 64 wavelengths) considered. Thus, heuristic scheme is an effective solution for adjusting burst length and hence lower burst loss probability.

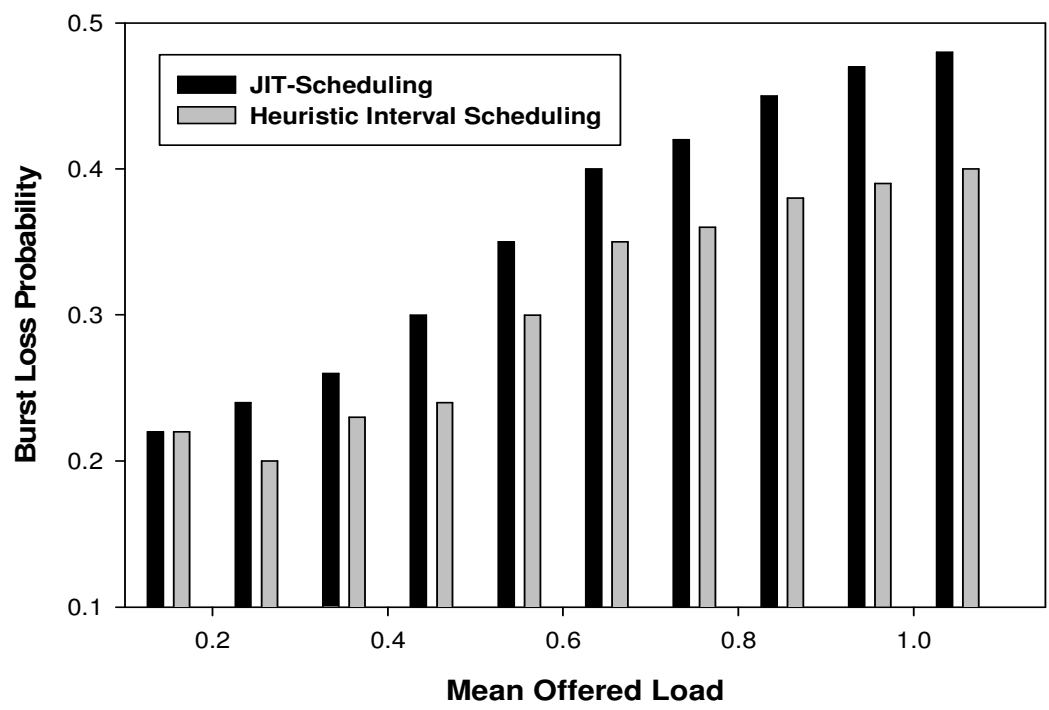

Fig.4: Burst loss probability for exponential inter-arrival time and burst length

\section{BURST ASSEMBLY}

In the Optical Burst Switching (OBS) Network, the burst assembly technique is one of the challenging issues in the implementation of the system. It has the influence on the burst characteristic, which gives an impact on the network performance. Burst assembly is the process of assembling incoming data from the higher layer into bursts at the ingress edge node of the OBS network. The burst assembly mechanism must then place these packets into bursts based on some assembly policy.

Since OBS is based on statistical multiplexing, burst contentions may arise at the nodes and thus resulting packet loss. Therefore, in order to make statistical multiplexing more efficient and also to reduce packet loss probability due to burst contentions, excessive variation in burst size, there is a need to investigate efficient burst assembly techniques. The following is the brief description of conventional burst assembly schemes.

(a)THRESHOLD-BASED WITHOUT TIME LIMITATION (TH)

This threshold-based assembly scheme uses the fixed burst size decision value. It does not include the maximum assembly delay time limitation, thus it does not guarantee for the maximum assembly delay time. The burst will be transmitted when its length reaches the threshold value. In case of constant packet size, the generated bursts will have the fixed size value. While packet size varies, the generated bursts will have a varied burst size value.

(b)TIMERS-BASED WITH MINIMUM BURST LENGTH (TM) 
The timer-counter-based burst assembly method is based on maximum waiting time and minimum burst length. The burst will be generated as soon as the assembly waiting time reaches the maximum time limitation. The size of the bursts must be equal to or bigger than a given minimum burst size. If the waiting time is reached while the burst size is still smaller than the minimum burst size, the burst will be padded to the minimum burst size and transmitted into the network.

(c)FIXED LENGTH THRESHOLD-BASED WITH TIME LIMITATION (FL)

A fixed length threshold-based assembly method uses both fixed threshold and time-out interval for constant burst size and limited delay time of the assembly function. The timer starts to count as soon as the first packet arrives at an assembly queue. The burst consisting of IP packets in the assembly queue is sent out whenever the time-out occurs or the number of packets in assembly queue reaches the threshold value. The additional technique, called padding, is used to make the generated burst size equal to the designed fixed value.

In order to alleviate an excessive variation in burst size, a novel burst assembly scheme named as AdaptiveThreshold with Fixed Maximum Time Limitation Burst Assembly (ATH-FMTL) has been proposed [9], which uses optimal burst length threshold and fixed maximum time limitation as the condition for burst generation. The burst length thresholds are increased or decreased in case the burst queue size, at the time of burst generation, is larger than upper threshold or smaller than lower threshold, respectively. The performance of proposed scheme is compared under the standard drop policy (DP) and the segmentation policy (SDP) for packet loss probability.

The simulation results show that the performance of the proposed Adaptive-Threshold with Fixed Maximum Time Limitation (ATH-FMTL) burst assembly scheme is better than conventional burst assembly schemes in terms of loss probability and average assembly delay. Also, the proposed scheme avoids a sudden increase in the burst size and makes the burst sent out smoother as compared to conventional schemes.

Figure 5 shows the average assembly delay time of four assembly methods in the network level. It can be seen that the system performance in terms of loss probability and average assembly delay time of each assembly method is clearly different in the low offered load condition but behave equally in high load circumstances. Even though the threshold-based method provides the lowest loss probability value, it also produces the highest average assembly delay in low load condition. This high assembly delay time is obtained because there is no time limitation in the threshold-based technique. In the other three techniques, which include the maximum delay time in their assembly function, the ATH-FMTL scheme provides the lowest loss probability and assembly delay time among these three techniques. It can be concluded that the adaptable threshold probability based assembly technique reduces the system loss probability from the fixed-based technique. Even though the average loss probability values of all assembly schemes are in the same range, there is a very high loss probability variation on the time-based method, when compared to the others.

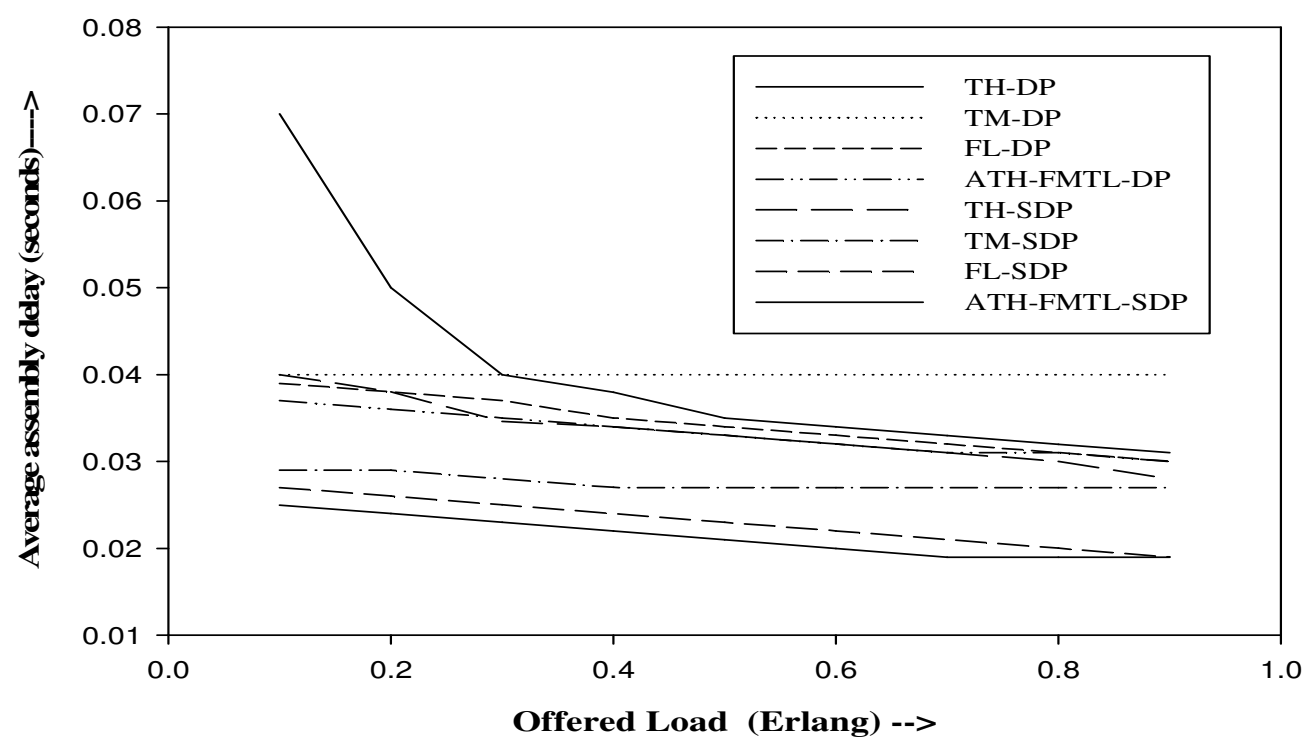


Fig. 5: Average assembly delay Vs offered load

\section{AN ADAPTIVE RESERVATION SCHEME FOR OPTICAL NETWORK}

In [10], the various Optical Burst Switching (OBS) reservation schemes have been examined in order to reduce data loss caused by either channel scheduling or resources. A novel multi-service OBS edge node with synchronized bandwidth reservation mechanism (SRM) has been proposed, which enables high speed network transport nodes to dynamically reserve bandwidth needed for active data burst flows. The performance of the proposed mechanism is evaluated by means of NS-2 simulation. The results show that the packet delay is kept within the constraint for each traffic flow and the performance metrics such as burst loss rate, throughput and fairness are remarkably improved.

Figure 6 shows the burst loss rate of conventional-OBS reservation and the proposed SRM-OBS method as a function of the offered traffic load per ingress OBS node and the length of burst flows. Comparing the burst loss rate with proposed SRM-OBS method, it is shown that the burst loss rate of the SRM is markedly improved. As the flow length increases from 50 bursts to 400 bursts in the SRM mechanism, the burst loss rate greatly decreases. At the beginning of a newly arriving burst flow, the bursts comprising the flow may experience burst collision for the slot reservation. Once core nodes recognize the periodicity of bursts and execute the SRM reservation for the subsequent bursts of the flow, the subsequent bursts do not suffer blocking. Consequently, the overall burst loss rate of long-lived burst flows is relatively lower than that of short-lived burst flows.

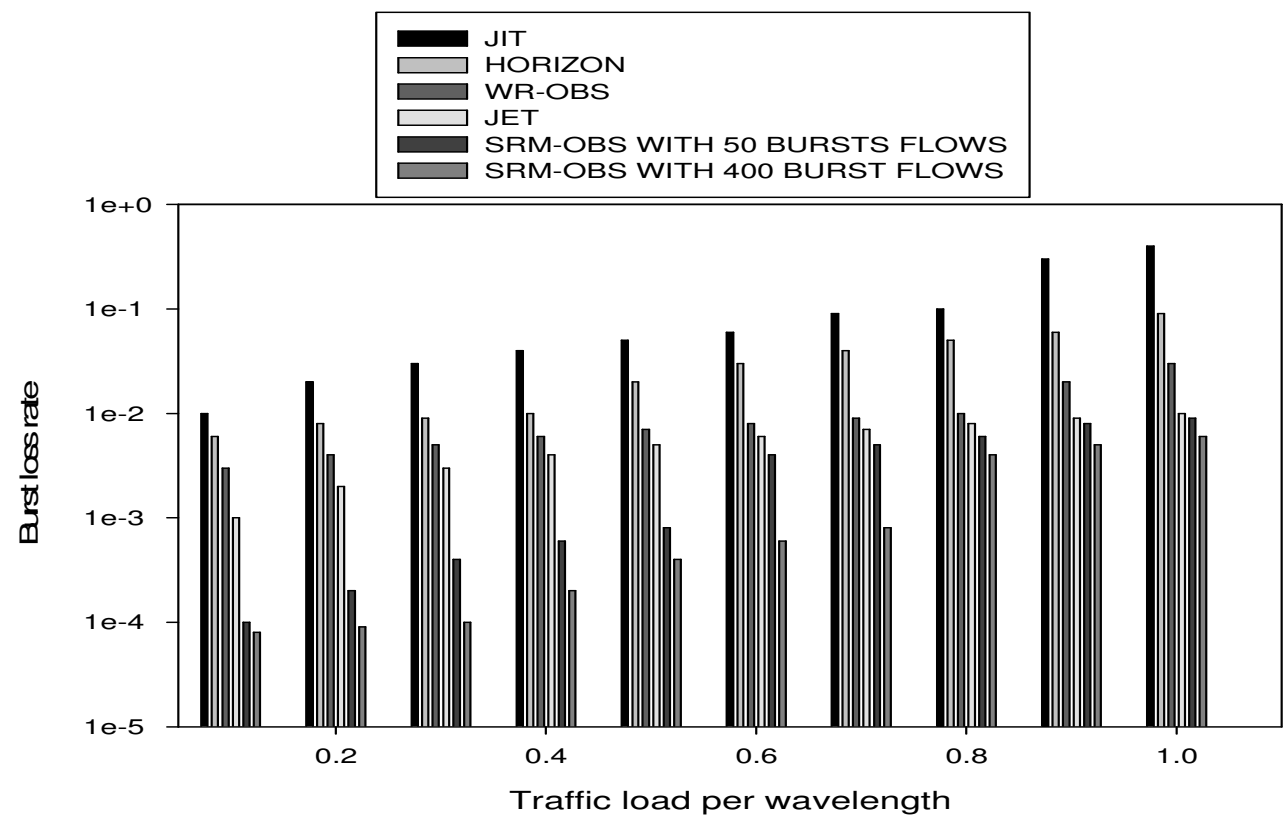

Fig.6: Burst loss rate vs. offered load with various flow lengths (wavelengths $=3$, reservations $=3$ bursts, loss notification $=2$ burst)

\section{TECHNIQUE FOR RESOLVING BURST CONTENTION}

In [11], in order to resolve burst contention (burst dropping), a new scheme for an optical burst switching (OBS) network has been proposed which is based on the integration of wavelength selection and burst assignment. The proposed scheme provides proportional differentiated services in buffer-less OBS networks by dynamically assigning more and longer periods of wavelengths to higher priority classes. In addition, burst head packets (BHPs) are buffered electrically so that fiber delay lines are unnecessary at core nodes. As a result, BHP of a lower priority class is buffered at the core router when it cannot find an available wavelength. It has an 
opportunity to reschedule its burst to the wavelengths that have been assigned to higher priority classes but have not yet been reserved. The proposed integrated scheme not only provides proportional differentiated services but also achieves lower average dropping probability without any preemption or segmentation mechanisms. Compared with existing approaches, the proposed

integrated scheme does not need to generate any special packets and needs to maintain only a few parameters at core nodes. The proposed scheme has several attractive features such as fast, simple implementation and improved burst dropping performance. Extensive simulation results have been used to demonstrate the effectiveness of the proposed scheme in comparison to traditional schemes.

Figures 7 and 8 illustrate the average burst loss ratio and normalized throughput at each link. Normalized throughput $=$ throughput/link capacity. Because each burst does not have the same size, the burst loss ratio differs from the bit loss ratio. Thus, the normalized throughput can be used to evaluate the network performance from another angle than burst loss ratio. The proposed integrated scheme has the highest normalized throughput and the lowest average burst loss ratio, whereas the traditional burst dropping scheme has the lowest normalized throughput and highest loss ratio at all load levels.

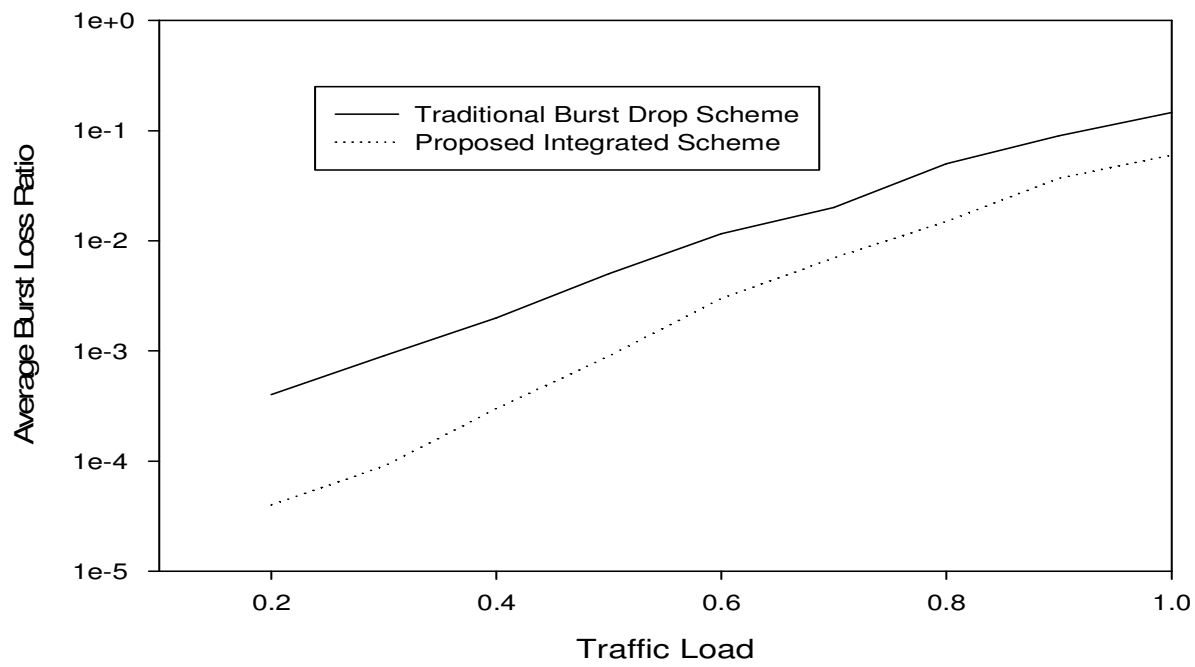

Fig.7: Average burst loss ratios for traditional burst drop and proposed integrated schemes

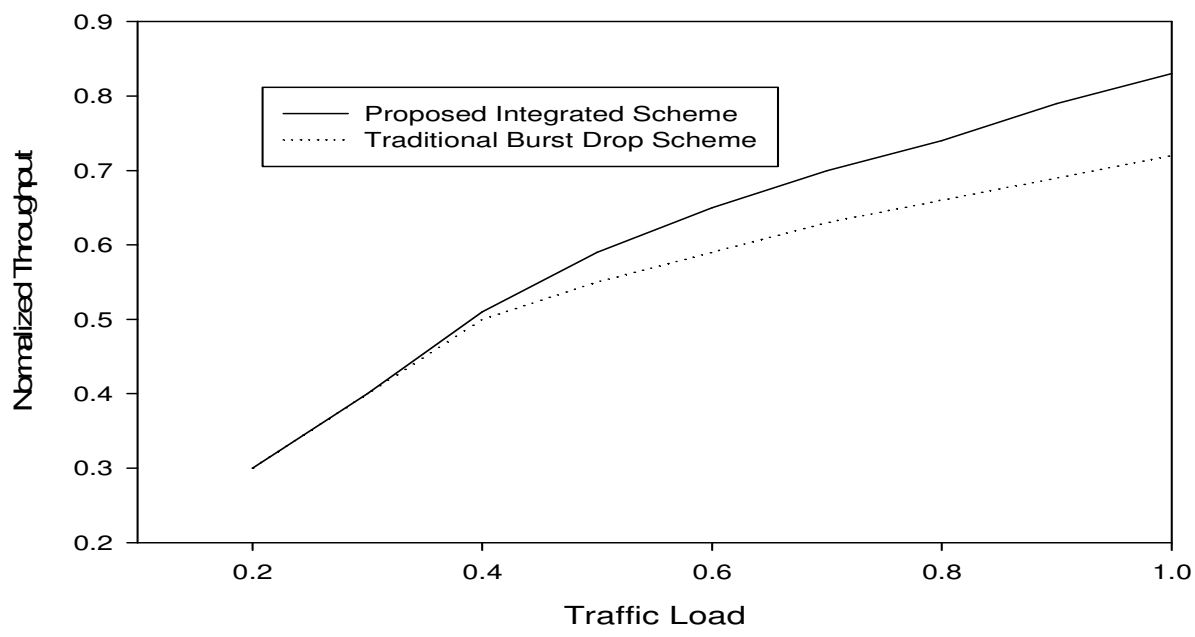

Fig.8: Normalized throughput for traditional burst drop and proposed integrated schemes 


\section{FLEXIBLE AND ENHANCING BANDWIDTH UTILIZATION BURST DROPPING}

Optical burst switching (OBS) has been proposed as a competitive hybrid switching technology to support the next generation optical Internet. However, due to their one-way resource reservation mechanism, OBS networks experience high bursts (thus packets) loss rate. In OBS networks, the contention is resolved either by dropping one of the contending bursts or more efficiently by dropping from one of the contending bursts only the parts that overlap with the other bursts. In both situations, only one data source will suffer the data loss in favor to the other. In [12], a new burst flexible and enhancing bandwidth utilization burst dropping technique has been proposed for contention resolution in optical burst switched networks. When contention occurs, any part of a contending burst (at random) could be dropped, instead of only the head or tail of bursts. The proposed dropping scheme makes bandwidth utilization more efficient and flexible. Simulation results show that the proposed dropping scheme performs better than existing burst dropping schemes.

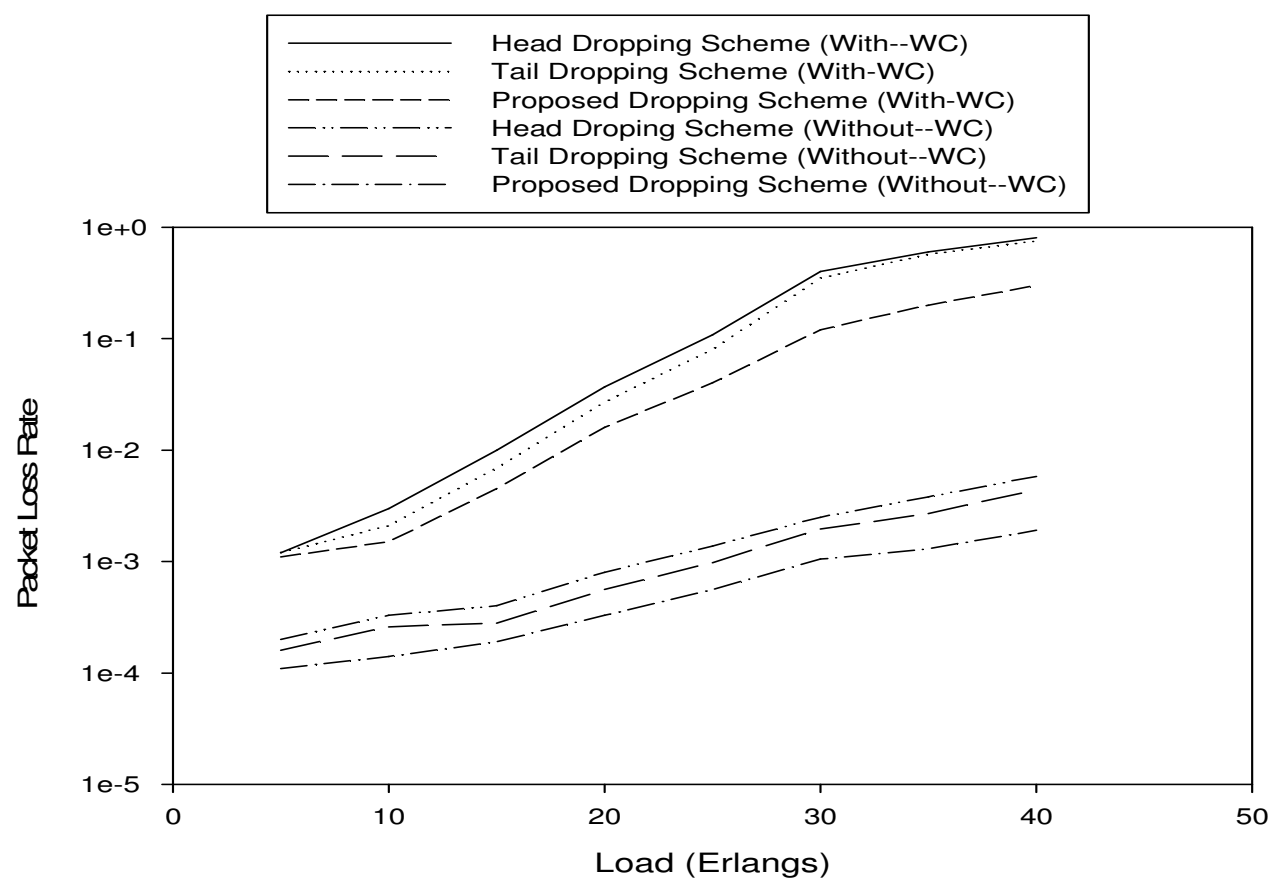

Fig. 9: Packet loss rate versus load for different burst dropping schemes [With wavelength converters (With-WC), without wavelength converters (Without-WC)]

Figure 9 plots the packet loss rate versus offered load for reference network with and without wavelength converters at every node. It is seen that when the load is light, the performances of the dropping policies look similar with wavelength converters, because contentions are not likely to happen with light load. However, if the load get heavier, the heavier the load is, the better the proposed dropping scheme performs compared to tail dropping and head dropping while there are not much difference between tail dropping and head dropping. Further, it is observed that even when the load is light, proposed dropping scheme out-performs tail dropping and head dropping because there are no wavelength converters and there are much more chances for contentions to happen even with light load.

\section{CONCLUSIONS}


In this paper, some of the performance oriented possible and optimal OBS solutions have been presented and described to make it a viable technology for all optical WDM networks. Based on the properties of optical burst flows and bandwidths provisioning in the networks, optimal solutions have been discussed related to OBS network architecture employing optical label processing; efficient scheduling and reservation schemes for optimizing channel and thus bandwidth utilization; optimal burst dropping schemes to provide differentiated service in order to support the quality of service (QoS) requirements of different applications; burst assembly schemes respect to burst loss probability; resource-reservation to alleviate contention in optical burst switching networks. By using simulation and analytical modeling, it is shown that the better results are obtained with the presented solutions such as lower blocking probability, gain in processing time, significant differentiation with regard to burst loss, minimized re-configuration and enhancement in bandwidth utilization, improvement in wavelength usage, throughput is thus increased and the network resources are used more effectively.

Based upon the above results, it is concluded that with respect to the current state of the technology, OBS combines the best features of both circuit switching and packet switching and thus acts as a viable technology for the next generation all optical networks.

\section{REFERENCES}

[1]T. Battestilli and H. Perros, “An introduction to optical burst switching”, IEEE Communications Magazine, Vol.41, No. 8, pp. S10 S15, August 2003.

[2]Y. Chen, C. Qiao, and X. Yu, “Optical Burst Switching: A New Area in Optical Networking Research,” IEEE Network Magazine, pp. 16-23, 2004

[3]J. Xu, C. Qiao, J. Li, G. Xu, "Efficient channel scheduling in optical burst switched networks", Proceedings of the IEEE INFOCOM, 2003.

[4]J Xu, C. Qiao, J. Li, G. Xu, "Efficient burst scheduling algorithms in optical burst-switched networks using geometric techniques", IEEE Journal on Selected Areas in Communication, Vol. 22, No. 9,pp. 1796-1881, November 2004.

[5]Li Yang, "Congestion control and quality-of-service (QoS) provisions for optical burst switched networks", Ph.D. Thesis, North Carolina State University, Raleigh, NC, May 2006.

[6]H. Kong, C. Philips, "Pre-booking reservation mechanism for next-generation optical networks", IEEE J. Sel. Top. Quantum Electron, Vol.12, No.4, pp. 645-652, 2006.

[7] Amit Kumar Garg et al., "A Novel Optical Burst Switching Architecture for High Speed Networks”, Chinese Optics Letters (Journal of Optical Society of America), Vol.6, No.11, pp.807-811, November 10, 2008.

[8] Amit Kumar Garg et al., "An Efficient Scheme for Optimizing Channel Utilization in OBS Networks", International Journal for Light and Electron Optics, Elsevier Science, Vol. 121, No. 9, pp.793-799, May 2010.

[9] Amit Kumar Garg et al., "Analysis of Burst/Packet Assembly Techniques in High Speed Optical Switching Network", Optik International Journal for Light and Electron Optics, Elsevier Science, Vol.122, No.7, pp.616-619, 2011.

[10] Amit Kumar Garg et al., "An adaptive reservation scheme for optical network", Optik - International Journal for Light and Electron Optics, Elsevier Science, Vol.122, No.4, pp.281-286, 2011.

[11] Amit Kumar Garg et al., "A Novel Technique to Resolve Burst Contention in OBS Networks", International Journal of Electronics, Taylor \& Francis, Vol.97, No.3, pp.295-308, March 2010.

[12]Amit Kumar Garg et al., "A new flexible and enhancing bandwidth utilization burst dropping technique for an OBS network", Optik International Journal for Light and Electron Optics, Elsevier Science, Vol.122, No.3, pp.225-227, 2011. 